\title{
THE POSITIVE MASS THEOREM NEAR NULL INFINITY
}

\author{
XIAO ZHANG
}

\begin{abstract}
In this short paper, we review recent progress on the positive mass theorem for spacelike hypersurfaces which approach to null infinity in asymptotically flat spacetimes. We use it to prove, if the functions $c(u, \theta, \psi), d(u, \theta, \psi)$ vanish at certain retarded time in vacuum Bondi's radiating spacetimes, then the Bondi mass is nonnegative up to that time.
\end{abstract}

\section{INTRODUCTION}

The definition of the total energy-momentum at spatial infinity was given by Arnowitt-Desser-Misnerfor in asymptotically flat spacetimes [1. The positivity of the ADM mass was proved by Schoen and Yau in a nontrivial isolated physical system which satisfies the dominant energy condition [16, 17. 18. Later it was proved by Witten using spinors [22]. The positive mass theorem plays a fundamental role in general relativity. It indicates the existence of the ground state in gravity.

Related to the gravitational radiation, there is a positive mass conjecture at null infinity. Physically, it is believed when gravitational radiation occurs, the energy of the system will be carried away by gravitational waves. Most physical systems have a conserved stress-energy tensor with a positive timelike component. Therefore they cannot radiate away more energy than they have initially. However, the gravitational field does not have a well-defined stress-energy tensor. An isolated gravitational system with finite ADM total energy-momentum might be able to radiate arbitrarily large amounts of energy. That it cannot happen is known as the positive mass conjecture at null infinity.

It is not available yet to set this conjecture in a mathematical rigorous way. In the pioneering work of Bondi, van der Burg, Metzner and Sachs on the gravitational waves in vacuum Bondi's radiating spacetimes, the Bondi mass associated to each null cone is defined and it is always non-increasing with respect to the retarded time 3, 15, 20. Therefore, the Bondi mass can be interpreted as the total mass of the isolated physical system measured after the loss due to the gravitational radiation up to that time. In this case, the positive mass conjecture at null infinity is equivalent to the positivity of Bondi mass.

Research is partially supported by National Natural Science Foundation of China under grant No. 10231050 and the Innovation Project of Chinese Academy of Sciences. 
The proof of the positivity of the Bondi mass was outlined by Schoen and Yau by modifying their arguments in the proof of the positivity of the ADM mass [19], and by physicists by applying Witten's spinor method, eg, see [10, 17, 2, 11, 14, 8. However, it needs to fill out all mathematical details.

The paper is organized as follows: In Section 2, we review Schoen-Yau's positive mass theorem at spatial infinity. In Section 3, we review author's positive mass theorem near null infinity. In Section 4, we review HuangYau-Zhang's theorem concerning the positivity of the Bondi mass in Bondi's radiating spacetimes.

\section{The POSITIVE MASS THEOREM AT SPATIAL INFINITY}

A spacetime is a 4-dimensional Lorentzian manifold $\left(\mathbf{L}^{3,1}, \mathbf{g}\right)$ which satisfies the Einstein field equations

$$
\mathbf{R}_{\alpha \beta}-\frac{\mathbf{R}}{2} \mathbf{g}_{\alpha \beta}=\mathbf{T}_{\alpha \beta},
$$

where $\mathbf{R}_{\alpha \beta}$ is the Ricci curvature of $\mathbf{g}$ and $\mathbf{R}$ is the scalar curvature of $\mathbf{g}$, $\mathbf{T}_{\alpha \beta}$ is the energy-momentum tensor.

There are some exact solutions of the vacuum field equations (i.e., $\mathbf{T}_{\alpha \beta}=$ $0)$ in polar coordinates $(r, \theta, \psi)$ where $0<r<\infty, 0 \leq \theta<\pi, 0 \leq \psi<2 \pi$ :

- Minkowski spacetime

$$
\mathbf{g}_{\text {Mink }}=-d t^{2}+d r^{2}+r^{2}\left(d \theta^{2}+\sin ^{2} \theta d \phi^{2}\right) ;
$$

- Schwarzschild spacetime

$$
\mathbf{g}_{S c h}=-\left(1-\frac{2 m}{r}\right) d t^{2}+\frac{d r^{2}}{1-\frac{2 m}{r}}+r^{2}\left(d \theta^{2}+\sin ^{2} \theta d \phi^{2}\right)
$$

- Kerr spacetime

$$
\begin{aligned}
\mathbf{g}_{\text {Kerr }}= & -\left(1-\frac{2 m r}{\Sigma}\right) d t^{2}-\frac{4 m a r \sin ^{2} \theta}{\Sigma} d t d \phi+\frac{\Sigma}{\triangle} d r^{2} \\
& +\Sigma d \theta^{2}+\left(r^{2}+a^{2}+\frac{2 m r a^{2} \sin ^{2} \theta}{\Sigma^{2}}\right) \sin ^{2} \theta d \phi^{2}
\end{aligned}
$$

where $\Sigma \equiv r^{2}+a^{2} \cos ^{2} \theta, \triangle \equiv r^{2}-2 m r+a^{2}$.

The parameters $m$ is the total mass, $a$ is the angular momentum per unit mass.

Let $(M, g, h)$ be a spacelike hypersurface in $\mathbf{L}^{3,1}, M$ is a 3 -dimensional manifold, $g$ is the Riemannian metric of $M$ and $h$ is the second fundamental form of $M$. It is usually called an initial data set. $(M, g, h)$ is asymptotically flat if there is a compact set $K \subset M$ such that $M \backslash K$ is the disjoint union of a finite number of subsets $M_{1}, \cdots, M_{l}$ - called the "ends" of $M$ - each 
diffeomorphic to $\mathbb{R}^{3} \backslash B_{r}$, where $B_{r}$ is the closed ball of radius $r$ with center at the coordinate origin. In each end, $g, h$ satisfy

$g_{i j}=\delta_{i j}+O\left(\frac{1}{r}\right), \partial_{k} g_{i j}=O\left(\frac{1}{r^{2}}\right), \partial_{l} \partial_{k} g_{i j}=O\left(\frac{1}{r^{3}}\right), h_{i j}=O\left(\frac{1}{r^{2}}\right), \partial_{k} h_{i j}=O\left(\frac{1}{r^{3}}\right)$

where $\left\{x^{i}\right\}$ is the Euclidean coordinates of $\mathbb{R}^{3}$.

The total energy $E_{l}$ and the total linear momentum $P_{l k}$ of end $M_{l}$ are defined by

$$
\begin{aligned}
E_{l} & =\frac{1}{16 \pi} \lim _{r \rightarrow \infty} \int_{S_{r, l}}\left(\partial_{j} g_{i j}-\partial_{i} g_{j j}\right) * d x^{i}, \\
P_{l k} & =\frac{1}{8 \pi} \lim _{r \rightarrow \infty} \int_{S_{r, l}}\left(h_{k i}-g_{k i} h_{j j}\right) * d x^{i},
\end{aligned}
$$

where $S_{r, l}$ is the sphere of radius $r$ in end $M_{l} \subset \mathbb{R}^{3}, 1 \leq k \leq 3$.

The spacetime $\left(\mathbf{L}^{3,1}, \mathbf{g}\right)$ satisfies the dominant energy condition if, for any timelike vector $W$,

(i) $\mathbf{T}_{u v} W^{u} W^{v} \geq 0$;

(ii) $\mathbf{T}^{u v} W_{u}$ is a non-spacelike vector.

Restricted on $\left(M^{3}, g, h\right)$, it implies that

$$
\frac{1}{2}\left(R+\left(h_{i}{ }_{i}\right)^{2}-h_{i j} h^{i j}\right) \geq \sqrt{\sum_{1 \leq i \leq 3}\left(\nabla^{j} h_{i j}-\nabla_{i} h^{j}{ }_{j}\right)^{2}}
$$

where $R$ is the scalar curvature of $g$.

Theorem 2.1. (The Schoen-Yau's positive mass theorem [16, 17, 18, 22]) If the spacetime $\left(\mathbf{L}^{3,1}, \mathbf{g}\right)$ satisfies the dominant energy condition, then, for asymptotically flat initial data set $(M, g, h)$,

$$
E_{l} \geq \sqrt{\sum_{1 \leq k \leq 3} P_{l k}^{2}}
$$

for each end $M_{l}$. Equality implies that $M$ has only one end and $\mathbf{L}^{3,1}$ is flat along $M$

In 1999, the author generalized the positive mass theorem to the spacetimes including the total angular momentum [23. The idea is to prove a positive mass theorem for a nonsymmetric initial data set $(M, g, p)$ where $p$ is a 2-tensor which is not necessarily symmetric.

\section{The POSITIVE MASS THEOREM NEAR NULL INFINITY}

In spacetimes, null hypersurfaces consist of null geodesics. Along null hypersurfaces, the induced metrics degenerate and the geometric properties are difficult to study. However, we can choose certain spacelike hypersurfaces 
to approach null infinity and use them to study null infinity. In Minkowski spacetime, the spacelike hypersurface

$$
t=\sqrt{1+r^{2}}
$$

has the hyperbolic metric $\breve{g}$ and the nontrivial second form $\breve{h}$

$$
\begin{aligned}
\breve{g} & =\frac{d r^{2}}{1+r^{2}}+r^{2}\left(d \theta^{2}+\sin ^{2} \theta d \psi^{2}\right), \\
\breve{h} & =\frac{d r^{2}}{1+r^{2}}+r^{2}\left(d \theta^{2}+\sin ^{2} \theta d \psi^{2}\right) .
\end{aligned}
$$

Denote the associated orthonormal frame $\left\{\breve{e}_{i}\right\}$ and coframe $\left\{\breve{e}^{i}\right\}$ by $\breve{e}_{1}=$ $\sqrt{1+r^{2}} \frac{\partial}{\partial r}, \breve{e}_{2}=\frac{1}{r} \frac{\partial}{\partial \theta}, \breve{e}_{3}=\frac{1}{r \sin \theta} \frac{\partial}{\partial \psi}, \breve{e}^{1}=\frac{d r}{\sqrt{1+r^{2}}}, \breve{e}^{2}=r d \theta, \breve{e}^{3}=r \sin \theta d \psi$. Denote $\breve{\nabla}$ the Levi-Civita connection of $\breve{g}$ and $\breve{\nabla}_{\breve{e}_{i}}$ by $\breve{\nabla}_{i}$. Based on the above model, we can define asymptotically null initial data set $(M, g, p)$ ( $p$ is not necessarily symmetric) of order $\tau$ if there is a compact set $K \subset M$ such that $M \backslash K$ is the disjoint union of a finite number of subsets $M_{1}, \cdots, M_{l}$ - called the "ends" of $M$ - each diffeomorphic to $\mathbb{R}^{3} \backslash B_{r}$, where $B_{r}$ is the closed ball of radius $r$ with center at the coordinate origin. In each end, the metric $g$ and the 2-tensor $p$ are $g\left(\breve{e}_{i}, \breve{e}_{j}\right)=\breve{g}\left(\breve{e}_{i}, \breve{e}_{j}\right)+a_{i j}, p\left(\breve{e}_{i}, \breve{e}_{j}\right)=\breve{p}\left(\breve{e}_{i}, \breve{e}_{j}\right)+b_{i j}$ where $a_{i j}$ and $b_{i j}$ satisfy

$a_{i j}=O\left(\frac{1}{r^{\tau}}\right), \breve{\nabla}_{k} a_{i j}=O\left(\frac{1}{r^{\tau}}\right), \breve{\nabla}_{k} \breve{\nabla}_{l} a_{i j}=O\left(\frac{1}{r^{\tau}}\right), b_{i j}=O\left(\frac{1}{r^{\tau}}\right), \breve{\nabla}_{k} b_{i j}=O\left(\frac{1}{r^{\tau}}\right)$.

Denote

$$
\begin{aligned}
\mathcal{E} & =\breve{\nabla}^{j} a_{1 j}-\breve{\nabla}_{1} \operatorname{tr}_{\breve{g}}(a)-\left(a_{11}-g_{11} \operatorname{tr}_{\breve{g}}(a)\right), \\
\mathcal{P}_{k} & =b_{k 1}-g_{k 1} \operatorname{tr}_{\breve{g}}(b) .
\end{aligned}
$$

The total energy and the total linear momentum of an asymptotically null initial data set $(M, g, p)$ on end $M_{l}$ are

$$
\begin{aligned}
E_{l \nu} & =\frac{1}{16 \pi} \lim _{r \rightarrow \infty} \int_{S_{r}} \mathcal{E} n^{\nu} r \breve{\omega}_{2} \wedge \breve{\omega}_{3}, \\
P_{l \nu, k} & =\frac{1}{8 \pi} \lim _{r \rightarrow \infty} \int_{S_{r}} \mathcal{P}_{k} n^{\nu} r \breve{\omega}_{2} \wedge \breve{\omega}_{3},
\end{aligned}
$$

where $S_{r, l}$ is the sphere of radius $r$ in end $M_{l} \subset \mathbb{R}^{3}, \nu=0,1,2,3, k=1,2,3$.

Theorem 3.1. (The positive mass theorem near null infinity 25]) Let ( $M, g, p)$ be a 3-dimensional asymptotically null initial data set of order $\tau=3$. Let

$$
\begin{aligned}
\mu & =\frac{1}{2}\left(R+\left(p_{i}^{i}\right)^{2}-p_{i j} p^{i j}\right), \\
\varpi_{j} & =\nabla^{i} p_{j i}-\nabla_{j} p_{i}^{i} \\
\sigma_{j} & =2 \nabla^{i}\left(p_{i j}-p_{j i}\right)
\end{aligned}
$$


where $R$ is the scalar curvature of $g$. If the initial data set satisfies the dominant energy condition

$$
\mu \geq \max \left\{\sqrt{\sum_{1 \leq j \leq 3} \varpi_{j}^{2}}, \sqrt{\sum_{1 \leq j \leq 3}\left(\varpi_{j}+\sigma_{j}\right)^{2}}\right\},
$$

then,

$$
E_{l 0}-P_{l 0,1} \geq \sqrt{\sum_{1 \leq i \leq 3}\left(E_{l i}-P_{l i, 1}\right)^{2}} .
$$

If equality holds, then $M$ has only one end and

$$
R_{i j k l}+p_{i k} p_{j l}-p_{i l} p_{j k}=0, \quad \nabla_{i} p_{j k}-\nabla_{j} p_{i k}=0, \quad \nabla^{j}\left(p_{i j}-p_{j i}\right)=0 .
$$

Remark 3.1. The proof of Theorem 3.1 is also valid for the case $\tau>\frac{3}{2}$ and the $E_{l \nu}-P_{l \nu, 1}$ are finite for $\nu=0,1,2,3$.

Remark 3.2. Theorem 3.1 and its application to the positivity of the Bondi mass (Remark 5.1 in [25]) were basically proved in November 2002 in early version of [25. The final revised version of [25] was sent to Chruściel on June 27, 2003. On July 23, 2003, the author received the preprint [5] from Chruściel. Translating into our formulation, they proved the same positive mass theorem as Theorem 3.1 for 3-dimensional initial data set $(M, g, K)$ in [5], where $g$ satisfies integrable condition (3.7) in [5], and $K$ satisfies

$$
K^{i j}=\frac{1}{r^{3}} L^{i j}+\frac{t r_{g} K}{3} g^{i j}, t r_{g} K=C+O\left(\frac{1}{r^{2}}\right)
$$

for certain trace-free tensor $L^{i j}$ and constant $C$.

\section{Bondi's RADIATING SPACETIMES}

Bondi's radiating spacetimes are vacuum spacetimes equipped with the following metric

$$
\begin{aligned}
\mathbf{g}_{\text {Bondi }}= & \left(\frac{V}{r} e^{2 \beta}+r^{2} e^{2 \gamma} U^{2} \cosh 2 \delta+r^{2} e^{-2 \gamma} W^{2} \cosh 2 \delta\right. \\
& \left.+2 r^{2} U W \sinh 2 \delta\right) d u^{2}-2 e^{2 \beta} d u d r \\
& -2 r^{2}\left(e^{2 \gamma} U \cosh 2 \delta+W \sinh 2 \delta\right) d u d \theta \\
& -2 r^{2}\left(e^{-2 \gamma} W \cosh 2 \delta+U \sinh 2 \delta\right) \sin \theta d u d \psi \\
& +r^{2}\left(e^{2 \gamma} \cosh 2 \delta d \theta^{2}+e^{-2 \gamma} \cosh 2 \delta \sin ^{2} \theta d \psi^{2}\right. \\
& +2 \sinh 2 \delta \sin \theta d \theta d \psi)
\end{aligned}
$$

where parameters $r>0,0 \leq \theta<\pi, 0 \leq \psi<2 \pi$ and $\beta, \gamma, \delta, U, V, W$ are smooth functions of

$$
x^{0}=u, \quad x^{1}=r, x^{2}=\theta, \quad x^{3}=\psi .
$$


The parameter $u$ is called the retarded coordinate. Physically, $u=$ constant requires to be null hypersurfaces. In Schwarzschild spacetime, the retarded coordinate $u=t-r-2 m \ln |r-2 m|$, and the metric is written also as

$$
\mathbf{g}_{S c h}=-\left(1-\frac{2 m}{r}\right) d u^{2}-2 d u d r+r^{2}\left(d \theta^{2}+\sin ^{2} \theta d \psi^{2}\right)
$$

The metric (4.1) was studied by Bondi, van der Burg, Metzner and Sachs in the theory of gravitational waves in general relativity [3, 15, 20]. They proved that the following asymptotic behavior holds for $r$ sufficiently large if the spacetime satisfies the outgoing radiation condition [20]

$$
\begin{aligned}
\gamma & =\frac{c(u, \theta, \psi)}{r}+\frac{C(u, \theta, \psi)-\frac{1}{6} c^{3}-\frac{3}{2} c d^{2}}{r^{3}}+O\left(\frac{1}{r^{4}}\right), \\
\delta & =\frac{d(u, \theta, \psi)}{r}+\frac{H(u, \theta, \psi)+\frac{1}{2} c^{2} d-\frac{1}{6} d^{3}}{r^{3}}+O\left(\frac{1}{r^{4}}\right), \\
\beta & =-\frac{c^{2}+d^{2}}{4 r^{2}}+O\left(\frac{1}{r^{4}}\right), \\
U & =-\frac{l(u, \theta, \psi)}{r^{2}}+\frac{p(u, \theta, \psi)}{r^{3}}+O\left(\frac{1}{r^{4}}\right), \\
W & =-\frac{\bar{l}(u, \theta, \psi)}{r^{2}}+\frac{\bar{p}(u, \theta, \psi)}{r^{3}}+O\left(\frac{1}{r^{4}}\right), \\
V & =-r+2 M(u, \theta, \psi)+O\left(\frac{1}{r}\right),
\end{aligned}
$$

where

$$
\begin{aligned}
l= & c_{, 2}+2 c \cot \theta+d_{, 3} \csc \theta \\
\bar{l}= & d_{, 2}+2 d \cot \theta-c_{, 3} \csc \theta \\
p= & 2 N(u, \theta, \psi)+3\left(c c_{, 2}+d d_{, 2}\right)+4\left(c^{2}+d^{2}\right) \cot \theta \\
& -2\left(c_{, 3} d-c d_{, 3}\right) \csc \theta \\
\bar{p}= & 2 P(u, \theta, \psi)+2\left(c_{, 2} d-c d_{, 2}\right)+3\left(c c_{, 3}+d d_{, 3}\right) \csc \theta .
\end{aligned}
$$

Under these conditions, the Bondi's radiating metric $\mathbf{g}_{B o n d i}$ is

$$
\begin{aligned}
& -\left(1-\frac{2 M}{r}+O\left(\frac{1}{r^{2}}\right)\right) d u^{2}-2\left(1-\frac{c^{2}+d^{2}}{4 r^{2}}+O\left(\frac{1}{r^{4}}\right)\right) d u d r \\
& +2\left(l+\frac{2 c l+2 d \bar{l}}{r}+O\left(\frac{1}{r^{2}}\right)\right) d u d \theta+2\left(\bar{l}-\frac{2 c \bar{l}-2 d l}{r}+O\left(\frac{1}{r^{2}}\right)\right) \sin \theta d u d \psi \\
& +r^{2}\left(1+\frac{2 c}{r}+O\left(\frac{1}{r^{2}}\right)\right) d \theta^{2}+r^{2}\left(1-\frac{2 c}{r}+O\left(\frac{1}{r^{2}}\right)\right) \sin ^{2} \theta d \psi^{2} \\
& +r^{2}\left(\frac{4 d}{r}+O\left(\frac{1}{r^{2}}\right)\right) \sin \theta d \theta d \psi .
\end{aligned}
$$

Since $\frac{\partial u}{\partial r} \neq 0$ in general, the metric (4.1) is not asymptotically flat at spatial infinity. We assume 
Condition A: Each of the six functions $\beta, \gamma, \delta, U, V, W$ and its derivatives up to the second orders have the same values at $\psi=0$ and $\psi=2 \pi$.

Condition B: For all $u, \theta_{0}=0$, or $\pi$,

$$
\int_{0}^{2 \pi} c\left(u, \theta_{0}, \psi\right) d \psi=0
$$

The Bondi energy-momentum of $u_{0}$-slice is defined as

$$
m_{\nu}\left(u_{0}\right)=\frac{1}{4 \pi} \int_{S^{2}} M\left(u_{0}, \theta, \psi\right) n^{\nu} d S
$$

for $\nu=0,1,2,3$, where

$$
n^{0}=1, \quad n^{1}=\sin \theta \cos \psi, \quad n^{2}=\sin \theta \sin \psi, \quad n^{3}=\cos \theta .
$$

The Bondi energy-momentum is the total energy-momentum measured after the loss due to the gravitational radiation up to that time.

In 1962, Bondi proved that the $m_{0}(u)$ is a non-increasing function of $u$ 3], i.e., more and more energy is radiated away.

Proposition 4.1. (Huang-Yau-Zhang 9]) Let $\left(\mathbf{L}^{3,1}, \mathbf{g}_{\text {Bondi }}\right)$ be a vacuum Bondi's radiating spacetime with metric $\mathbf{g}_{\text {Bondi }}$ given by (4.1). Suppose that Condition $\mathbf{A}$ and Condition $\mathbf{B}$ hold. Then

$$
\frac{d}{d u}\left(m_{0}-\sqrt{\sum_{1 \leq i \leq 3} m_{i}^{2}}\right) \leq 0 .
$$

Proof : Denote $|m|=\sqrt{m_{1}^{2}+m_{2}^{2}+m_{3}^{2}}$. We assume $|m| \neq 0$ otherwise it reduces to the Bondi mass-loss formula. We have

$$
\begin{aligned}
\frac{d}{d u}\left(m_{0}-|m|\right)= & -\frac{1}{4 \pi}\left[\int_{S^{2}}\left(\left(c_{, 0}\right)^{2}+\left(d_{, 0}\right)^{2}\right) d S\right. \\
& \left.-\frac{1}{|m|} \sum_{1 \leq i \leq 3} m_{i} \int_{S^{2}}\left(\left(c_{, 0}\right)^{2}+\left(d_{, 0}\right)^{2}\right) n^{i} d S\right] .
\end{aligned}
$$

Using $\left(n^{1}\right)^{2}+\left(n^{2}\right)^{2}+\left(n^{3}\right)^{2}=1$ and Hölder inequality, we obtain

$$
\sum_{1 \leq i \leq 3}\left[\int_{S^{2}}\left(\left(c_{, 0}\right)^{2}+\left(d_{, 0}\right)^{2}\right) n^{i} d S\right]^{2} \leq\left[\int_{S^{2}}\left(\left(c_{, 0}\right)^{2}+\left(d_{, 0}\right)^{2}\right) d S\right]^{2} .
$$

It together with Cauchy-Schwarz inequality implies

$$
\sum_{1 \leq i \leq 3} m_{i} \int_{S^{2}}\left(\left(c_{, 0}\right)^{2}+\left(d_{, 0}\right)^{2}\right) n^{i} d S \leq|m| \int_{S^{2}}\left(\left(c_{, 0}\right)^{2}+\left(d_{, 0}\right)^{2}\right) d S .
$$

Therefore (4.2) holds.

Q.E.D. 
We study the asymptotically null initial data set $(M, g, h)$ where $M$ is given by

$$
u=\sqrt{1+r^{2}}-r+\frac{\left(c^{2}+d^{2}\right)_{u=0}}{12 r^{3}}+\frac{a_{3}(\theta, \psi)}{r^{4}}+a_{4}
$$

where $a_{4}(r, \theta, \psi)$ is a smooth function which satisfies: In the Euclidean coordinate systems $\left\{z^{i}\right\},|z|=r, a_{4}=o\left(\frac{1}{r^{4}}\right), \partial a_{4}=o\left(\frac{1}{r^{5}}\right), \partial \partial a_{4}=o\left(\frac{1}{r^{6}}\right)$ as $r \rightarrow \infty$. We compute the induced metric $g$ and the second fundamental form $h$. Define $a \approx b$ if and only if $a=b+o\left(\frac{1}{r^{3}}\right)$.

$$
\begin{aligned}
& g\left(\breve{e}_{1}, \breve{e}_{1}\right) \approx 1+\left.\frac{16 a_{3}+M-c c_{, 0}-d d_{, 0}}{2 r^{3}}\right|_{u=0}, \\
& g\left(\breve{e}_{1}, \breve{e}_{2}\right) \approx-\frac{l}{2 r^{2}}+\frac{12 N-3 l_{, 0}+4\left(c c_{, 2}+d d_{, 2}\right)}{12 r^{3}}, \\
& g\left(\breve{e}_{1}, \breve{e}_{3}\right) \approx-\frac{\bar{l}}{2 r^{2}}+\frac{12 P-3 \bar{l}_{, 0}+4 \csc \theta\left(c c, 3+d d_{, 3}\right)}{12 r^{3}}, \\
& g\left(\breve{e}_{2}, \breve{e}_{2}\right) \approx 1+\frac{2 c}{r}+\frac{2\left(c^{2}+d^{2}\right)+c_{, 0}}{r^{2}} \\
& +\frac{c^{3}+c d^{2}+2 C+2\left(c c_{, 0}+d d_{, 0}\right)+\frac{c, 00}{4}}{r^{3}}, \\
& g\left(\breve{e}_{2}, \breve{e}_{3}\right) \approx \frac{2 d}{r}+\frac{d_{, 0}}{r^{2}}+\frac{c^{2} d+d^{3}+2 H+\frac{d_{, 00}}{4}}{r^{3}} \\
& g\left(\breve{e}_{3}, \breve{e}_{3}\right) \approx 1-\frac{2 c}{r}+\frac{2\left(c^{2}+d^{2}\right)-c_{, 0}}{r^{2}} \\
& +\frac{-c^{3}-c d^{2}-2 C+2\left(c c_{, 0}+d d_{, 0}\right)-\frac{c, 00}{4}}{r^{3}}, \\
& h\left(\breve{e}_{1}, \breve{e}_{1}\right) \approx 1+\frac{c^{2}+d^{2}}{r^{2}}+\frac{16 a_{3}-M}{r^{3}}, \\
& h\left(\breve{e}_{1}, \breve{e}_{2}\right) \approx \frac{l}{2 r^{2}}+\frac{1}{2 r^{3}}\left[\frac{l_{, 0}}{2}-2\left(c^{2}+d^{2}\right) \cot \theta-4 N\right. \\
& \left.\left(-c d_{, 3}+c_{, 3} d\right) \csc \theta-\frac{13}{3}\left(c c_{, 2}+d d_{, 2}\right)\right], \\
& h\left(\breve{e}_{1}, \breve{e}_{3}\right) \approx \frac{\bar{l}}{2 r^{2}}+\frac{1}{2 r^{3}}\left[\frac{\bar{l}_{, 0}}{2}+c d_{, 2}-c_{, 2} d-4 P\right. \\
& \left.-\frac{13}{3}\left(c c, 3+d d_{, 3}\right) \csc \theta\right] \text {, } \\
& h\left(\breve{e}_{2}, \breve{e}_{2}\right) \approx 1+\frac{c}{r}+\frac{c_{, 0}}{r^{2}}+\frac{1}{4 r^{3}}\left[3 M-16 a_{3}-4 C-2 l_{, 2}\right. \\
& \left.-2 c\left(c^{2}+d^{2}\right)+5\left(c c_{, 0}+d d_{, 0}\right)+\frac{3}{2} c_{, 00}\right], \\
& h\left(\breve{e}_{2}, \breve{e}_{3}\right) \approx \frac{d}{r}+\frac{d_{, 0}}{r^{2}}+\frac{1}{4 r^{3}}\left[-2 d\left(c^{2}+d^{2}\right)+2 d \cot ^{2} \theta\right. \\
& +2 d \csc ^{2} \theta-4 c_{, 3} \cot \theta \csc \theta-d_{, 33} \csc ^{2} \theta
\end{aligned}
$$




$$
\begin{aligned}
& \left.-d_{, 2} \cot \theta-d_{, 22}-4 H+\frac{3}{2} d_{, 00}\right], \\
h\left(\breve{e}_{3}, \breve{e}_{3}\right) \approx & 1-\frac{c}{r}-\frac{c_{, 0}}{r^{2}}+\frac{1}{4 r^{3}}\left[3 M-16 a_{3}+4 C\right. \\
+ & 2 c\left(c^{2}+d^{2}\right)+5\left(c c_{, 0}+d d_{, 0}\right)-\frac{3}{2} c_{, 00} \\
& \left.-2 l \cot \theta-2 \bar{l}_{, 3} \csc \theta\right] .
\end{aligned}
$$

Here all functions in the right hand sides take value at $u=0$ and all derivatives with respect to $x^{2}$ and $x^{3}$ are taken after substituting $u=0$.

Replacing $u$ by $u-u_{0}$, applying Theorem 3.1. Remark 3.1 to $(M, g, h)$ and using (4.2), we can prove the following theorem concerning positivity of the Bondi mass.

Theorem 4.1. (Huang-Yau-Zhang [9] Let $\left(\mathbf{L}^{3,1}, \mathbf{g}_{\text {Bondi }}\right)$ be a vacuum Bondi's radiating spacetime with metric $\mathbf{g}_{\text {Bondi }}$ given by [4.1). Suppose that Condition A and Condition B hold. If there is $u_{0}$ such that $\left.c\right|_{u=u_{0}}=\left.d\right|_{u=u_{0}}=0$, then

$$
m_{0}(u) \geq \sqrt{\sum_{1 \leq i \leq 3} m_{i}^{2}(u)}
$$

for all $u \leq u_{0}$. Moreover, if the equality holds for all $u \leq u_{0}, \mathbf{L}^{3,1}$ is flat in the region $u \leq u_{0}$.

It turns out the Bondi mass cannot become negative if

$$
\lim _{u \rightarrow \infty} c(u, \theta, \psi)=\lim _{u \rightarrow \infty} d(u, \theta, \psi)=0 .
$$

In 9], we are also studying the positivity of the Bondi mass by using SchoenYau's argument in 19 .

\section{REFERENCES}

[1] S. Arnowitt, S. Deser, C. Misner, Coordinate invariance and energy expressions in general relativity, Phys. Rev. 122(1961), 997-1006.

[2] A. Ashtekar, G. Horowitz, Energy-momentum of isolated systems cannot be null, Phys. Lett. 89A(1982), 181-184.

[3] H. Bondi, H. van der Burg, A. Metzner, Gravitational waves in general relativity VII. Waves from axi-symmetric isolated systems, Proc. Roy. Soc. London A 269(1962), 21-52.

[4] P. Chruściel, M. Herzlich, The mass of asymptotically hyperbolic Riemannian manifolds, Pacific J. Math., 212(2003), 231-264.

[5] P. Chruściel, J. Jezierski, S. Leski, The Trautman-Bondi mass of initial data sets, Adv. Theor. Math. Phys. 8(2004), 83-139; gr-qc/0307109

[6] P. Chruściel, G. Nagy, The mass of spacelike hypersurfaces in asymptotically anti-de Sitter space-times, Adv. Theor. Math. Phys. 5(2001), 697-754.

[7] G. Horowitz, M. Perry, Gravitational energy cannot become negative, Phys. Rev. Lett. 48(1982), 371-374. 
[8] G. Horowitz, P. Tod, A relation between local and total energy in general relativity, Commun. Math. Phys. 85(1982), 429-447.

[9] W.-l. Huang, S.T. Yau, X. Zhang, Positivity of the Bondi mass in Bondi's radiating spacetimes, In preparation.

[10] W. Israel, J. Nester, Positivity of the Bondi gravitational mass, Phys. Lett. 85A(1981), 259-260.

[11] M. Ludvigsen, J. Vickers, A simple proof of the positivity of the Bondi mass, J. Phys. A: Math. Gen. 15(1982), L67-L70.

[12] J. Nester, A new gravitational energy expression with a simple positivity proof, Phys. Lett. 83A(1981), 241-242.

[13] T. Parker, C. Taubes, On Witten's proof of the positive energy theorem, Commun. Math. Phys. 84(1982), 223-238.

[14] O. Reula, K. Tod, Positivity of the Bondi energy, J. Math. Phys. 25(1984), 1004-1008.

[15] R. Sachs, Gravitational waves in general relativity VIII. Waves in asymptotically flat space-time, Proc. Roy. Soc. London, A 270(1962), 103-126.

[16] R. Schoen, S.T. Yau, On the proof of the positive mass conjecture in general relativity, Commun. Math. Phys. 65(1979), 45-76.

[17] R. Schoen, S.T. Yau, The energy and the linear momentum of spacetimes in general relativity, Commun. Math. Phys. 79(1981), 47-51.

[18] R. Schoen, S.T. Yau, Proof of the positive mass theorem. II, Commun. Math. Phys. 79(1981), 231-260.

[19] R. Schoen, S.T. Yau, Proof that the Bondi mass is positive, Phys. Rev. Lett. 48(1982), 369-371.

[20] M. van der Burg, Gravitational waves in general relativity IX. Conserved quantities, Proc. Roy. Soc. London A 294(1966), 112-122.

[21] X. Wang, Mass for asymptotically hyperbolic manifolds, J. Diff. Geom. 57(2001), 273299.

[22] E. Witten, A new proof of the positive energy theorem, Commun. Math. Phys. 80(1981), 381-402.

[23] X. Zhang, Angular momentum and positive mass theorem, Commun. Math. Phys. 206(1999), 137-155.

[24] X. Zhang, Remarks on the total angular momentum in general relativity, Commun. Theore. Phys., 39(2003), 521-524.

[25] X. Zhang, A definition of total energy-momenta and the positive mass theorem on asymptotically hyperbolic 3-manifolds I, Commun. Math. Phys., 249(2004), 529-548.

Institute of Mathematics, Academy of Mathematics and System Sciences, Chinese Academy of Sciences, Beijing 100080, China

E-mail address: xzhang@amss.ac.cn 